\title{
Nanoscale
}

Check for updates

Cite this: Nanoscale, 2021, 13, 14866

\section{Extracellular vesicles from adipose stromal cells combined with a thermoresponsive hydrogel prevent esophageal stricture after extensive endoscopic submucosal dissection in a porcine model $\dagger$}

\author{
Elise Coffin, ${ }^{a}$ Alice Grangier, ${ }^{b}$ Guillaume Perrod, ${ }^{a}$ Max Piffoux, ${ }^{b}$ Iris Marangon, ${ }^{b}$ \\ Imane Boucenna, ${ }^{\mathrm{b}}$ Arthur Berger, (D) ${ }^{a}$ Leila M'Harzi, ${ }^{\mathrm{C}}$ Jessica Assouline, ${ }^{\mathrm{d}}$ \\ Thierry Lecomte, ${ }^{\mathrm{e}}$ Anna Chipont, ${ }^{\mathrm{f}}$ Coralie Guérin, ${ }^{f}$ Florence Gazeau, (D) ${ }^{\mathrm{b}}$ \\ Claire Wilhelm, (D) ${ }^{\mathrm{b}}$ Christophe Cellier, ${ }^{\mathrm{g}}$ Olivier Clément, ${ }^{\mathrm{h}}$ \\ Amanda Karine Andriola Silva (D)* $\star^{\mathrm{b}}$ and Gabriel Rahmi**a,g
}

\begin{abstract}
In this study, we investigated the combination of extracellular (nano) vesicles (EVs) from pig adipose tissue-derived stromal cells (ADSCs) and a thermoresponsive gel, Pluronic ${ }^{\circledR}$ F-127 (PF-127), to prevent stricture formation after endoscopic resection in a porcine model. ADSC EVs were produced at a liter scale by a high-yielding turbulence approach from ADSCs 3D cultured in bioreactors and characterized in terms of size, morphology and membrane markers. The thermoresponsive property of the PF-127 gel was assessed by rheology. The pro-regenerative potency of ADSC EVs was investigated ex vivo in esophageal biopsies under starvation. In vivo tests were performed in a porcine model after extended esophageal endoscopic mucosal dissection (ESD). Pigs were randomized into 3 groups: control $(n=6)$, gel $(n=6)$ or a combination of $1.45 \times 10^{12} \mathrm{EVs}+$ gel $(n=6)$. Application of gel \pm EVs was performed just after ESD with a follow-up finalized on day 21 post-ESD. There was a trend towards less feeding disorder in the EV + gel group in comparison with the gel and the control groups ( $16.67 \%$ vs. $66.7 \%$ vs. $83.33 \%$, respectively) but without reaching a statistically significant difference. A significant decrease in the esophageal stricture rate was confirmed by endoscopic, radiological and histological examination for the EV + gel group. A decrease in the mean fibrosis area and larger regenerated muscularis mucosae were observed for the EV + gel group. In summary, the application of EVs + gel after extended esophageal endoscopic resection succeeded in preventing stricture formation with an anti-fibrotic effect. This nano-therapy may be of interest to tackle an unmet medical need considering that esophageal stricture is the most challenging delayed complication after extended superficial cancer resection by endoscopy.
\end{abstract}

Received 24th February 2021 Accepted 7th July 2021 DOI: 10.1039/d1nr01240a rsc.li/nanoscale

\footnotetext{
${ }^{a}$ Laboratoire Imagerie de l'Angiogénèse, Plateforme d'Imagerie du Petit Animal, PARCC, INSERM U970, Laboratoire de Recherches Biochirugicales (Fondation Carpentier), Université de Paris, 56 rue Leblanc, 75015 Paris, France

${ }^{b}$ Laboratoire Matière et Systèmes Complexes (MSC), Université de Paris, UMR 7057 CNRS, 75205 Paris cedex 13, France.E-mail: amanda.silva@univ-paris-diderot.fr ${ }^{c}$ Department of Surgery, Hôpital Européen Georges Pompidou, Assistance Publique des Hôpitaux de Paris, Université de Paris, 20 rue Leblanc 75015, France ${ }^{d}$ Departement of Radiology, Hôpital Saint Louis, Assistance Publique des Hôpitaux de Paris, Université de Paris, 1 Avenue Claude Vellefaux, 75010 Paris, France ${ }^{e}$ CIRE Plateform, UMR 0085, Physiologie de la Reproduction et des comportements, INRA, Centre Val De Loire, 37380 Nouzilly, France
}

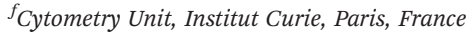 \\ ${ }^{g}$ Gastro-Enteroloy and Endoscopy Department, Hôpital Européen Georges Pompidou, \\ Assistance Publique des Hôpitaux de Paris, Université de Paris, 20 rue Leblanc \\ 75015, France. E-mail: gabriel.rahmi@aphp.fr \\ ${ }^{h}$ Department of Radiology, Hôpital Européen Georges Pompidou, Assistance Publique \\ des Hôpitaux de Paris, Université de Paris, 20 rue Leblanc 75015, France \\ $\dagger$ Electronic supplementary information (ESI) available: Supplementary figures. \\ See DOI: 10.1039/d1nr01240a \\ † These authors contributed equally to this work.
}




\section{Introduction}

Extracellular vesicles (EVs) are membrane-enclosed sub-cellular nano-entities, mainly from 50 to $300 \mathrm{~nm}$, that constitute a farreaching intercellular communication system. ${ }^{1}$ EVs, especially from stromal cells (SCs), are currently reported to contribute to tissue repair and homeostasis by modulating cell recruitment, proliferation, migration, and differentiation. ${ }^{2-4}$ ADSC EVs have been increasingly considered an alternative to cells for regenerative medicine in a paradigm shift with cell therapy evolving to sub-cellular (EV) therapy. ${ }^{5,6}$ Previous reports evidenced the beneficial effect of ADSC EVs in the therapy of liver, heart, kidney, skin and brain injuries. ${ }^{6-11}$ The SC EV therapy holds the promise to mitigate the risks of uncontrolled cell differentiation, replication, or vascular occlusion while offering storage and shelf-life gains. ${ }^{12,13}$ Besides, the immune-privileged status of SC EVs may enable us to consider "off-the-shelf" nanotherapy in an allogeneic approach. ${ }^{14,15}$

Due to their promising features, EVs may represent innovative biogenic nano-therapies for tackling the current unmet needs in regenerative medicine. In this regard, we focus herein on esophageal stricture, which is a complication following extensive endoscopic submucosal dissection for the management of esophageal cancer. Esophageal cancer is the 6th cause of malignancy-related death in the world. ${ }^{16}$ The high morbidity/mortality rate related to surgical resection ${ }^{17}$ prompted the development of minimally invasive techniques to remove superficial cancer by the endoscopic procedure. Endoscopic submucosal dissection (ESD) represents the most recent technique allowing en bloc resection regardless of the lesion size. ${ }^{18}$ Stricture rates after extensive esophageal ESD range from $66 \%$ to $100 \% .{ }^{19,20}$ Extensive ESD, defined by resection of more than $\frac{3}{4}$ of the esophageal circumference, is considered the main risk factor for esophageal stricture. Deep tumor invasion beyond the epithelium (i.e. T1a tumors according to the Japanese classification of superficial tumors) is the second main risk factor for a stricture occurrence. ${ }^{21}$

The pathogenesis of esophageal stricture is orchestrated by immune cells and myofibroblasts. The loss of the epithelium barrier function following ESD triggers immune cell recruitment, resulting in an infiltrating inflammatory process. ${ }^{22}$ In a later phase, fibroblasts and pericytes transform into $\alpha$-smooth actin-expressing myofibroblasts responsible for fibrosis. ${ }^{23}$ Fibrosis in the submucosa is related to muscularis mucosae destruction and muscularis propria infiltration, leading to esophageal strictures. ${ }^{24}$

In the case of esophageal stricture after extended ESD, the first-line therapy is endoscopic balloon dilations. Despite a high success rate, there are several limitations, listed as follows: (i) a high number of balloon dilations from 6 to 20, (ii) several procedures under general anesthesia, and (iii) the risk of severe complications such as bleeding, perforation and mediastinitis. ${ }^{25-27}$ There are no satisfactory therapies for postesophageal ESD stricture prevention. Oral, intravenous, and local injections of steroids were tested in this regard without satisfactory results in terms of stricture prevention, ${ }^{28}$ and severe adverse events have been reported such as abscesses. ${ }^{29}$ Different endoscopic shielding techniques have been investigated and failed to prevent esophageal strictures. ${ }^{30}$ Therefore, there is a strong need for an efficient esophageal stricture prevention therapy.

Regenerative medicine is a new promising field and may represent an alternative for stricture prevention therapy. For instance, a previous report showed promising results in terms of post-ESD stricture prevention after cell sheet transplantation using oral epithelial cells. ${ }^{31}$ Our team demonstrated that a double cell sheet of ADSCs allowed a reduction of esophageal stricture and fibrosis in a porcine model. ${ }^{32}$ In fact, SCs are competent to elicit immunosuppressive and anti-inflammatory effects, which may be mediated by a paracrine effect. ${ }^{33}$ In this regard, many recent studies have pointed out the role of EVs in this paracrine effect. ${ }^{34}$ Our aim herein was to investigate the effect of ADSC EV nano-therapy on the prevention of esophageal stricture.

To facilitate EV delivery into the esophagus, we used a thermoresponsive gel (Poloxamer 407 or Pluronic ${ }^{\circledR}$ F-127, PF-127) ${ }^{35}$ containing EVs. This thermoresponsive gel composed of nano-micelles is expected to allow minimally invasive injection in the liquid state at $<20{ }^{\circ} \mathrm{C}$ and gelation in situ at body temperature to favor $\mathrm{EV}$ residence time in the site of interest. ${ }^{36}$ Besides being a delivery vehicle, $\mathrm{PF}-127$ seems to be beneficial for wound healing by recruiting some factors like VEGF and TGF $\beta 1$ and increasing the density of micro-vessels, collagen level and rate of formation of granulation tissue. ${ }^{37}$ Our team previously evidenced the therapeutic effect of ADSC EVs and PF-127 gel for esophageal fistula healing in a porcine model. ${ }^{38}$ The aim of this study was to evaluate the effect of allogeneic porcine ADSC EVs in combination with the PF-127 gel to prevent esophageal strictures after an extensive ESD in a clinically relevant porcine model. ADSC EVs were produced at a liter scale by a high-yielding turbulence approach in bioreactors $^{38}$ from cells in 3D culture and administered with the gel by endoscopy in a minimally invasive strategy (Fig. 1). In this study, endoscopic, radiological and histological analyses were performed. The degree of esophageal stenosis on day 21 (D21) assessed by a blinded radiological examination was considered the primary endpoint.

\section{Experimental section}

\section{Production and characterization of thermoresponsive PF-127 gels}

Preparation of PF-127 gels. PF-127 was dissolved at a concentration of $20 \%$ (weight/weight) in a saline solution under stirring for 24 hours at $4{ }^{\circ} \mathrm{C}$. Then, sterilizing filtration $(0.22 \mu \mathrm{m}$ filter) was carried out at $4{ }^{\circ} \mathrm{C}$.

Characterization of PF-127 gels. Rheological studies were performed for the PF-127 hydrogels with a Physica RheoCompass MCR 302 (Anton Paar) using a cone and plate geometry $\left(\right.$ diameter $=50 \mathrm{~mm}$, cone angle $=1^{\circ}$ ) and a solvent trap to prevent evaporation. The measurements of $G^{\prime}$ and $G^{\prime \prime}$, 


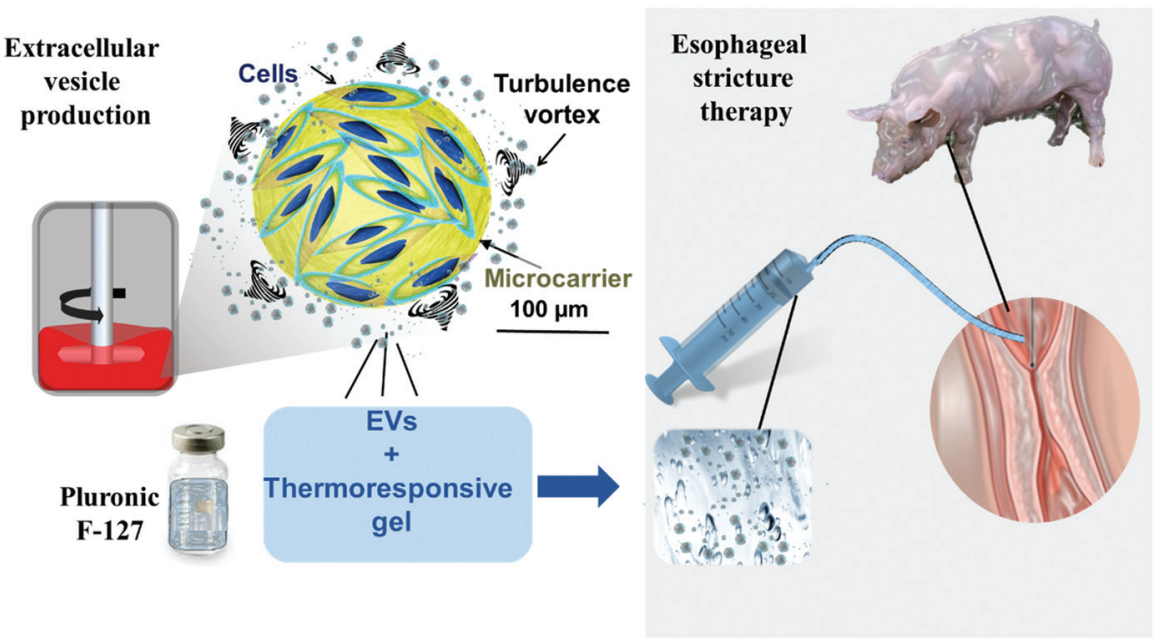

Fig. 1 Schematic representation of the experimental approach consisting of producing EVs by turbulence stimulation from swine ADSCs cultured on microcarriers in bioreactors. ADSC EVs were combined with the Pluronic F-127 thermoresponsive hydrogel and administered at $<19{ }^{\circ} \mathrm{C}$ (below the gel transition temperature) at post-resection esophageal lesion gelling in situ at body temperature.

the elastic and viscous moduli, respectively, as a function of temperature, were performed. The temperature was gradually increased by $5{ }^{\circ} \mathrm{C} \mathrm{min}^{-1}$ starting from $5{ }^{\circ} \mathrm{C}$ to $40^{\circ} \mathrm{C}$. The solgel transition temperature, cross-over between $G^{\prime}$ and $G^{\prime \prime}$, was investigated.

\section{ADSC culture, EV production and EV characterization}

ADSC culture in 2D flasks. EVs were produced from pig ADSCs (CD105+, CD90+, CD45-, CD31- from ABCell-Bio, Paris, France). First, ADSCs were grown in 2D flasks under standard culture conditions $\left(37^{\circ} \mathrm{C}\right.$ and $\left.5 \% \mathrm{CO}_{2}\right)$ in a culture medium comprising alpha modified Eagle's medium (alpha MEM), GlutaMax, $10 \%$ fetal bovine serum (FBS), and $1 \%$ penicillin and streptomycin (PS). When cells had reached about $80 \%$ confluence, they were detached with PBS and trypsin and then centrifuged for $5 \mathrm{~min}$ at $300 \mathrm{~g}$. After centrifugation, the supernatant was removed and the cells were resuspended in $50 \mathrm{ml}$ of complete medium.

ADSC EV production in 3D cell culture by turbulence stimulation. A 3D cell culture was performed in a $1 \mathrm{~L}$ spinner flask bioreactor containing Cytodex 3 beads of about $200 \mu \mathrm{m}$ diameter, to which complete alpha MEM GlutaMax culture medium was added. Cells were inoculated at a ratio of 5:1 cells per bead and subjected to 24 cycles of $45 \mathrm{~min}$, followed by $3 \mathrm{~min}$ of agitation within the bioreactor (at 30 rotations per minute) to allow a homogeneous distribution of cells on beads. Cells were left in culture until they were confluent (approximately 7 days) with a daily check of cell distribution on beads and cell density. In total, 30 to $70 \%$ of the culture medium was changed every 2-3 days according to the level of confluence. Four washes with serum-free DMEM were then performed, and then cells on beads were placed in serum-free DMEM $+1 \%$ PS without phenol red. The spinner flask bioreactor containing a magnetic impeller was then placed on a magnetic stirring plate. The stirring intensity was set to obtain a diameter of the smallest vortex of $35 \mu \mathrm{m}$ for 4 hours to generate turbulence shear stress stimulation. ${ }^{39}$ After that, cellular debris of the supernatant was removed by centrifugation at $2000 \mathrm{~g}$ for $10 \mathrm{~min}$. EVs were then ultracentrifuged at $110000 \mathrm{~g}$ for $1 \mathrm{~h}, 10 \mathrm{~min}$ to obtain an EV pellet.

Cell viability and detachment analyses. A Nucleocounter NC-200 (Chemometec A/S, Allerod, Denmark) device was used for cell viability and detachment analyses with calibrated cassettes from the supplier containing acridine orange and DAPI to quantify dead and live cells, respectively. The software NucleoView NC-200 version 1.3.0.0 was used. The cell viability and detachment on beads were determined following the manufacturer's instructions using the supplied A and B solutions for cell lysis. Briefly, all cells were lysed and stained with DAPI in the first cassette to count the total number of nuclei. Then, in the second cassette, only cells in the sample supernatant (after bead sedimentation) were stained with acridine orange and DAPI. Considering that all the cells on the beads are alive, the percentages of viable and detached cells were deduced.

Nanoparticle tracking analysis. The size distribution and concentration measurements of ADSC EVs were performed with a Nanosight (NS300, Malvern, UK). Before measurements, samples were diluted to the appropriate concentration (between $7 \times 10^{7}$ parts per $\mathrm{ml}$ and $5 \times 10^{8}$ parts per $\mathrm{ml}$ ) in sterile PBS. A total of 5 movies of $60 \mathrm{~s}$ each were recorded for each sample. The acquisition was performed with the following parameters: cameral level $=16$ and screen gain $=1$. Processing was performed at a gain of 10 and a detection threshold of 5 .

Cryo-transmission electron microscopy. A volume of 2-7 $\mu \mathrm{l}$ of ADSC EVs was transferred to an electron microscopy grid featuring a perforated carbon film coating (Ted Pella, Redding, CA, USA). The liquid in excess was blotted off with filter paper, and the grid was then quickly plunged into liquid ethane. Analysis was performed using a MET Jeol 2100 (LaB6) at $80 \mathrm{kV}$. 
Imaging flow cytometry. ADSC EVs were analyzed by flow cytometry imaging (Amnis $®$ ImageStream $₫$ ) using human anti-CD63 phycoerythrin (PE) and anti-CD81 allophycocyanin (APC) antibodies (BioLegend) and anti-CD9 fluorescein isothiocyanate (FITC) antibodies (CliniSciences). Briefly, 3.3 $\mu \mathrm{L}$ of anti-CD9 $(3.3 \mu \mathrm{l}$ of anti-CD63 and $2.5 \mu \mathrm{l}$ of anti-CD81) were mixed with $5 \mu \mathrm{L}$ of EVs $\left(1.6 \times 10^{12}\right.$ particles per $\left.\mathrm{mL}\right)$ in a final volume of $50 \mu \mathrm{l}$, completed with PBS. Each antibody was used alone before performing multiple staining. Incubation was carried out for $15 \mathrm{~min}$ in the dark. A total of 100000 events were analyzed. Images were collected in the bright field, dark field, a green channel for FITC, a yellow channel for PE and a red channel for APC fluorophores simultaneously. Speed beads were used for calibration $\left(\right.$ Amnis $^{\odot}{ }^{\circledR}$ SpeedBeads ${ }^{\odot}$ ). The analysis was first carried out by plotting the side scatter intensity (SSC) versus the fluorescence intensity corresponding to CD9 FITC, CD63 PE or CD81 APC. Gates corresponding to the apoptotic bodies (AB), large vesicles (lEVs) and small vesicles (sEV) were set according to their SSCs. In the second step, events in the SEV and IEV gates, for each antibody, were analyzed by plotting fluorescence intensities for CD9 FITC versus CD63 PE; CD81 APC versus CD9 FITC; and CD81 APC versus CD63 PE. Amnis IDEAS ${ }^{\odot}$ software was used for data analysis.

Ex vivo pro-survival tests in pig esophageal biopsies. The pro-survival effect of ADSC EVs on pig esophageal biopsies was evaluated ex vivo via an Alamar test. Pig esophageal biopsies were collected under general anesthesia $\left(10 \mathrm{mg} \mathrm{kg}^{-1}\right.$ intramuscular ketamine and $8 \mathrm{mg} \mathrm{kg}^{-1}$ intravenous propofol). A radial jaw was used to collect biopsies, which were then transferred to DMEM containing $100 \mathrm{U} \mathrm{mL}^{-1}$ penicillin and $100 \mathrm{U} \mathrm{mL}^{-1}$ streptomycin. Biopsies were individually placed in 96-well plates (one biopsy per well). The Alamar blue test (Invitrogen) was performed for all wells individually and used as the baseline. The test was carried out according to the supplier's instructions. Biopsies were then incubated for $72 \mathrm{~h}$ with ADSC EVs in a serum-free DMEM containing $2.5 \times 10^{10}$ or $1.25 \times 10^{11}$ EVs $\mathrm{mL}^{-1}$. The serum-free medium and complete medium were used as negative and positive controls, respectively. The Alamar test was carried out again at $24 \mathrm{~h}$ and $72 \mathrm{~h}$ time points. The obtained data were normalized for each biopsy individually using the baseline values.

In vivo studies. This protocol was conducted at the Biosurgical Research Laboratory of the Alain Carpentier Foundation in Paris and the National Institute for Agricultural Research (INRA) in Val de Loire Center. It was approved by the local ethics committee of Descartes University (no. 14099). A total of 18 female pigs between 30 and $50 \mathrm{~kg}$ were randomized into 3 groups: a control group $(n=6)$, a gel group $(n=6)$, and an EV + gel group $(n=6)$.

ESD technique. ESD under general anesthesia was performed on Do in all pigs. Animals received $2 \mathrm{mg} \mathrm{kg}^{-1}$ tiletamine-zolazepam and $4 \mathrm{mg} \mathrm{kg}^{-1}$ propofol IV for anesthesia induction. Anesthesia was maintained through $2 \%$ isoflurane. Animals were fasted 24 hours before the procedure for solid food and 8 hours before the procedure for liquid ingestion. ESD was performed in all pigs on D0 using a standard adult gastroscope (EG-530WR Fujifilm, Japan) and a Flushknife 1.5 BT (Fujifilm, Japan). An electrosurgical unit ERBE ICC 350 (ERBE Technology, Germany) and a pulsed pressure pump JW-2 (Fujifilm, Japan) were also used. After marking a virtual esophageal tumor, saline was injected into the sub-mucosa to facilitate dissection. The ESD strategy was to make a tunnel (proximal and distal incision and then submucosal dissection) $5 \mathrm{~cm}$ long and $\frac{3}{4}$ circumferential between 50 and $55 \mathrm{~cm}$ of the dental arch. The next step was lateral incision (left and right), and final dissection allowed a complete en bloc resection. All resected pieces were retrieved, pinned on a dedicated plate and measured.

PF-127 gel application. In the gel group, just after ESD, PF-127 (8 mL) was applied to the scar using an endoscopic plastic catheter (Liguory 7 Fr, Cook Medical, USA). PF-127 was injected in the liquid form at a temperature $<19^{\circ} \mathrm{C}$. In order to prevent gel transition and catheter clogging, the catheter was kept on ice. Gel transition took place in contact with the lesion at body temperature.

ADSC EV application with PF-127 gel. EVs stored in a $-80{ }^{\circ} \mathrm{C}$ freezer were thawed and diluted in saline or PF-127 at $20 \%$ just before ESD. For EV administration, a total of $1.45 \times 10^{12}$ ADSC EVs were divided into three fractions and applied into the lesions after ESD in the following sequence:

(1) $1 / 3$ of EVs were dispersed in $10 \mathrm{~mL}$ of saline and sprayed on the scar with a spray catheter (Alton, China);

(2) $1 / 3$ of EVs were dispersed in $10 \mathrm{~mL}$ of saline and injected into the edges of the scar (needle Interject, Boston Scientific, USA);

(3) $1 / 3$ of EVs were dispersed in $8 \mathrm{~mL}$ of the gel and applied to uniformly cover the scar using a catheter (Liguory 7Fr, Cook Medical, USA).

PF-127 gel application

For the gel group, only $8 \mathrm{~mL}$ of PF-127 gel was applied uniformly to cover the scar using a catheter (Liguory 7Fr, Cook Medical, USA).

\section{Follow-up}

Clinical evaluation. Daily examination of the pigs was performed as follows: assessment of pain, behavior, food intake, regurgitation, and vomiting. Dysphagia was scored based on the Mellow-Pinkas score (0: normal swallowing, 1: unable to swallow a proportion of the solid diet, 2: able to swallow a semi-solid diet; 3: able to swallow liquids only, 4: complete dysphagia including saliva). ${ }^{40}$

Radiological evaluation. The primary endpoint was the degree of esophageal stenosis on D21 assessed by a blinded radiological examination. A fluoroscopy was performed (2 orthogonal incidences) using Arcadis (Siemens, Germany) with a contrast agent (Omnipaque 300, Guerbet, France) injected into the operator channel to perform esophagography. The most relevant radiological incidence (anteroposterior or lateral) was kept, and we calculated a degree of stenosis as follows: [ 1 - (length of the short axis inside stenosis/length of the normal axis under stenosis $) \times(100)]$. 
Endoscopic evaluation. Endoscopic evaluation was performed on D21. The following two criteria were noted: (i) the possibility to pass with the gastroscope (diameter of $9.2 \mathrm{~mm}$ ) through the esophageal lumen; and (ii) the esophageal lumen diameter estimated using close or open biopsy forceps (measuring respectively $2 \mathrm{~mm}$ and $7 \mathrm{~mm}$ ).

Histological evaluation. On D21, pigs were euthanized and the entire esophagus including the stricture area was surgically resected. Specimens were stored in $4 \%$ paraformaldehyde for 24 hours before changing the medium to $70 \%$ alcohol. Between 5 and 7 blocks from the stricture area were created and included in paraffin. Then, slides of $4 \mu \mathrm{m}$ thickness were stained using hematoxylin and eosin and Sirius red. Finally, all the slides were digitally scanned (Digitiser Hamamatsu Photonics $®$, France), and dedicated software

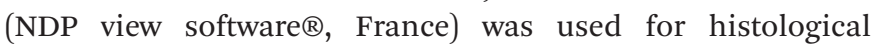
analysis.

The following criteria were measured: the maximal surface of fibrosis, maximal length of muscularis propria, and maximal length of defect of epithelium.

Statistical analysis. For comparison of qualitative variables between the different groups, an exact Fischer test was performed (expected values less than 5). Non-normal quantitative variables were compared using the non-parametric KruskalWallis test. A difference was considered statistically significant if $p<0.05$. If there was a significant overall difference, a Mann-Whitney test compared one group to another. Statistical analysis was performed using GraphPadPrism software (Graphpad Software, La Jolla, Calif., USA) for non-parametric Kruskal-Wallis tests, and Fisher's exact tests were performed via statistical software R.

\section{Results}

Characterization of viscoelastic properties of $\mathrm{PF}-127$ for the thermo-controlled EV delivery strategy

The PF-127 gel at $20 \%$ featured a transition temperature of $19.3{ }^{\circ} \mathrm{C}$ (Fig. 2A). At $24.7^{\circ} \mathrm{C}$, the storage modulus reached a plateau, imparting stability to the gel. At $37^{\circ} \mathrm{C}$, the elastic modulus was $2 \times 10^{4} \mathrm{~Pa}$ and the loss modulus was $9 \times 10^{2} \mathrm{~Pa}$. Therefore, at the body temperature of pigs, PF-127 is a gel and its viscoelastic properties are expected to confer good mechanical strength to be retained on the esophageal scar after endoscopic resection.

\section{Allogeneic ADSC EVs produced in high yield in bioreactors at a liter scale by the turbulence approach}

ADSCs were first cultured in 2D in T-flasks with a viability $>90 \%$ (ESI Fig. 1 and Table $1 \dagger$ ). In a scalable approach, cells were cultured in $3 \mathrm{D}$ on the surface of Cytodex 3 beads in $1 \mathrm{~L}$ spinner flask bioreactors with a viability of $>90 \%$ (ESI Fig. 2A$\mathrm{D}$ and Table $2 \dagger$ ). The EV production was triggered by turbulence stimulation by controlling the stirring speed of the bioreactor in order to reach a Kolmogorov length of $35 \mu \mathrm{m}$ in 4 hours. $^{39}$ The producer cell viability after turbulence stimulation was $>81 \%$ (ESI Fig. $2 \mathrm{E}-\mathrm{G}$ and Table $3 \dagger$ ).

The turbulence approach enabled the production of 62700 particles per ADSC in 4 hours, for instance. Starvation in $2 \mathrm{D}$ flasks for $72 \mathrm{~h}$ (with a post-production cell viability of $61.7 \%$ ) provided a particle yield of 14550 per cell, which was about 4 times less and 18 times longer (results not shown). ADSC EVs were isolated by centrifugation/ultracentrifugation steps and characterized by NTA (Fig. 2B and C). For instance, a total of 3
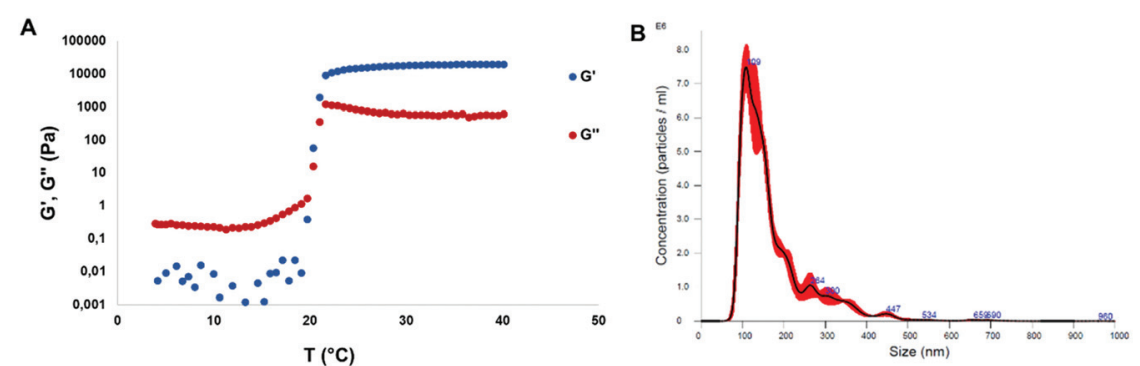

C

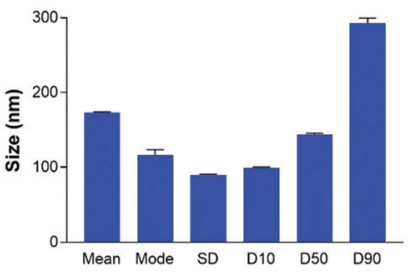

D

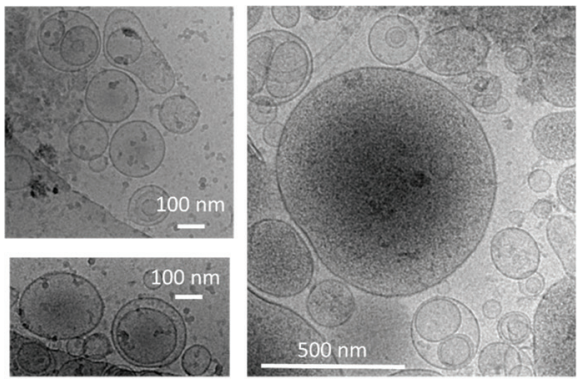

Fig. 2 Characterization of Pluronic F-127 by rheological analysis and ADSC EV characterization by nanoparticle tracking analysis and cryogenic transmission electron microscopy. Evolution of dynamic storage modulus $\left(G^{\prime}\right)$ and loss modulus $\left(G^{\prime \prime}\right)$ of Pluronic $\mathrm{F}-127$ at $20 \%$ and a heating rate of $5{ }^{\circ} \mathrm{C}$ $\mathrm{min}^{-1}$ from $5^{\circ} \mathrm{C}$ to $40^{\circ} \mathrm{C}$ for the determination of the sol-gel transition temperature ( $G$ ' and $G^{\prime \prime}$ cross over) (A). Size distribution of ADSC EVs, averaged on 5 recorded videos (B). Mean, mode, standard deviation and percentiles of the size of EV samples, represented as the mean \pm SEM of three independent batches by nanoparticle tracking analysis (C). Cryogenic transmission electron microscopy images of ADSC EVs, zoom $\times 10000$ (D). 
$\times 10^{12}$ EVs were obtained from 250 million ADSCs in $1 \mathrm{~L}$ bioreactors with a volume of $380 \mathrm{~mL}$ of medium after purification. The size distribution and concentration of EVs were analyzed by NTA. The sample featured characteristic EV size distribution that mainly ranged from 100 to $250 \mathrm{~nm}$. The mean size and mode were $172.8 \mathrm{~nm}$ and $116.3 \mathrm{~nm}$, respectively, with a size distribution standard deviation of $89.3 \mathrm{~nm}$. For this sample, $90 \%$ of particles were smaller than $293.1 \mathrm{~nm}, 50 \%$ of particles were smaller than $143.7 \mathrm{~nm}$, and $10 \%$ of particles were smaller than $99 \mathrm{~nm}$. Cryo-microscopy analysis showed characteristic round-shaped membrane-delimitated structures with high size polydispersity between 100 and $500 \mathrm{~nm}$ (Fig. 2D).

\section{Allogeneic ADSC EVs produced by turbulence featured classical EV markers according to image-based high- throughput multispectral analysis}

EVs were characterized using multispectral imaging flow cytometry (Fig. 3). This technique merges high-throughput flow cytometry with high statistical power for the visualization advantages of confocal microscopy on a single event basis. Images of 100000 events per sample were collected in the bright field, dark field, as well as in a green fluorescence channel for FITC (CD9 antibody detection), a yellow one for PE (CD63 antibody detection) and a red one for APC (CD81 antibody detection). Events were gated as small EVs (sEVs) and large EVs (lEVs) when plotting side scatter (SSC) intensity versus fluorescence intensity corresponding to CD9 FITC, CD63 PE or CD81 APC (Fig. 3A-C): 971, 314 and 1240 events, respectively. The majority of them were sEVs. For instance, sEVs represented more than $65 \%$ of the EV events concerning CD63 PE and more than $85 \%$ of the EV events concerning CD9 FITC or CD81 APC fluorescence intensities (Fig. 3D). Representative images of sEVs and lEVs (Fig. 3E and F, respectively) indicated some single- and double-positive events.

SEV and lEV gate events from the SSC versus CD9 FITC fluorescence intensity (Fig. 3A) were further plotted for CD81 APC versus CD63 PE fluorescence (Fig. 3G and $\mathrm{H}$, respectively) to evaluate single CD9+ (gate CD63-CD81-), double CD81+CD9+ only (gate CD63-CD81+), double CD63+CD9+ only (gate CD63+CD81-) and triple CD63+CD81+CD9+ (gate CD63+CD81+) populations. These biparametric dot plots and their bar graph representation (Fig. 3I) indicated that $\sim 92 \%$ of sEVs were single CD9+ and $\sim 7 \%$ of them were double CD63+CD9+.

Considering the reduced event number (314), EV gate events from SSC versus CD63 PE fluorescence intensity (Fig. 3B) were not further analyzed.

EV gate events from the SSC versus CD81 APC fluorescence intensity (Fig. 3C) were further plotted for CD63 PE versus CD9 FITC fluorescence (Fig. 3J and $\mathrm{K}$ for sEVs and lEVs, respectively) to evaluate single CD81+ (gate CD9-CD63-), double CD63+CD81+ only (gate CD9-CD63+), double CD9+CD81+ only (gate CD9+CD63-) and triple CD9+CD63+CD81+ (gate CD9+CD63+) populations. These biparametric dot plots and their bar graph representation (Fig. 3L) indicated that $~ 99 \%$ of SEV population was single CD81+ with a negligible double or triple positive population. Concerning lEVs, a negligible percentage of the population was CD9+CD81+ only, $\sim 8 \%$ were double CD63+CD81+ only, $\sim 26 \%$ were triple CD9+CD63+CD81+ and 65\% were single CD81+.

The apoptotic body $(\mathrm{AB})$ gate was constituted of events featuring important bright- and dark-field signals, but the events were devoid of fluorescence signals (ESI Fig. $3 \dagger$ ). Therefore, events in the $\mathrm{AB}$ gate were not considered as EVs herein.

\section{Allogeneic ADSC EVs produced by turbulence elicit pro- survival effect ex vivo on the pig esophageal biopsies under starvation}

The cell survival of the pig esophageal biopsies under starvation for 24 and $72 \mathrm{~h}$ incubated with or without ADSC EVs was tested by the Alamar blue assay. The pig esophageal biopsies incubated in complete medium and serum-depleted medium (starvation) were used as positive and negative controls, respectively. This test was carried out on a single biopsy basis with one biopsy per well (Fig. 4A). Cell survival was assessed at T0, T24 and T72 h. Normalization to the T0 value was performed to reduce inter-biopsy variability. At $24 \mathrm{~h}$ incubation, ADSC EVs slightly increased the biopsy survival under starvation compared to the negative control (Fig. 4B). At $72 \mathrm{~h}$ incubation, ADSC EVs significantly increased the biopsy survival at the highest dose of $1.25 \times 10^{11} \mathrm{EVs} \mathrm{mL}^{-1}$ compared to the negative control (Fig. 4C).

\section{Allogeneic ADSC EVs produced by turbulence combined with the PF-127 gel prevent stricture formation with an anti-fibrotic effect following extended esophageal endoscopic resection in pigs}

Both ESD and therapy were carried out on D0, and in vivo evaluation was finalized on day 21 (Fig. 5). All ESDs were performed without complications. The mean procedure duration was $30 \pm$ 10 minutes. Pieces of dissection were removed and measured. The lesion's size was comparable between the three groups ( $p=$ 0.40 for the height of the piece and $p=0.75$ for the width).

\section{Clinical evaluation after ESD}

Eating disorders such as regurgitation and vomiting were observed from day 12 in 55.5\% of pigs. There was a trend towards a therapeutic benefit for the $\mathrm{EV}+$ gel group, but no significant difference was observed comparing control, gel and $\mathrm{EV}+$ gel groups (respectively, 83.33\%, 66.7, and 16.67\%, $p=$ 0.11). These results are shown in Table 1 . Dysphagia score means were respectively $2.66,2.50$, and 1.50 for control, gel and $\mathrm{EV}+$ gel groups.

\section{Endoscopic evaluation at D21}

The passage of the gastroscope through esophageal stricture was possible for only one pig, and it was in the EV + gel group. The passage of the closed biopsies forceps ( $2 \mathrm{~mm}$ in diameter) through the esophageal stricture was possible in all cases. The passage of open forceps ( $7 \mathrm{~mm}$ diameter) was more frequently feasible in the $\mathrm{EV}+$ gel group compared to the gel and control ones $(66.7 \%$ vs. $33.3 \%$ and $0 \%, p=0.08)$ (Fig. 6A and Table 1 ). 

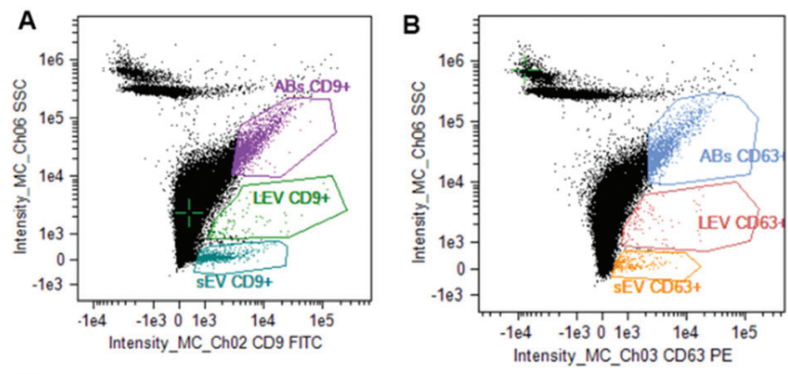

E

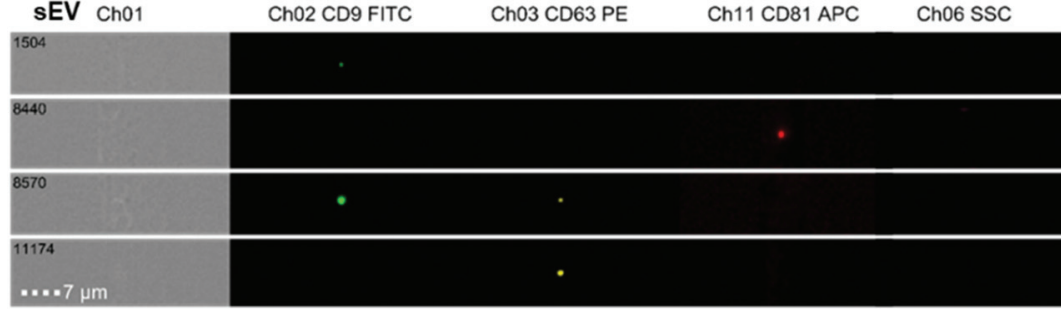

$\mathbf{F}$
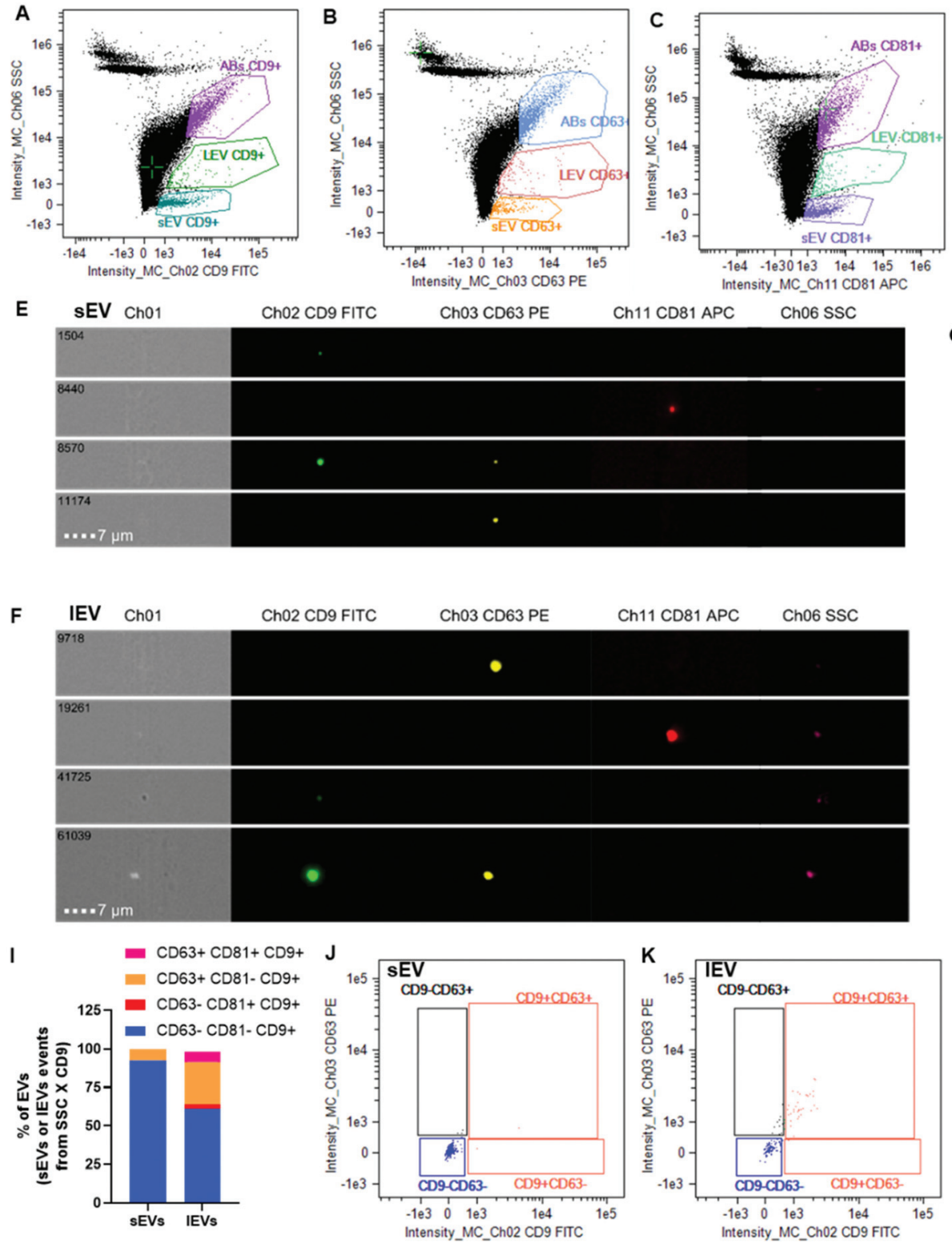
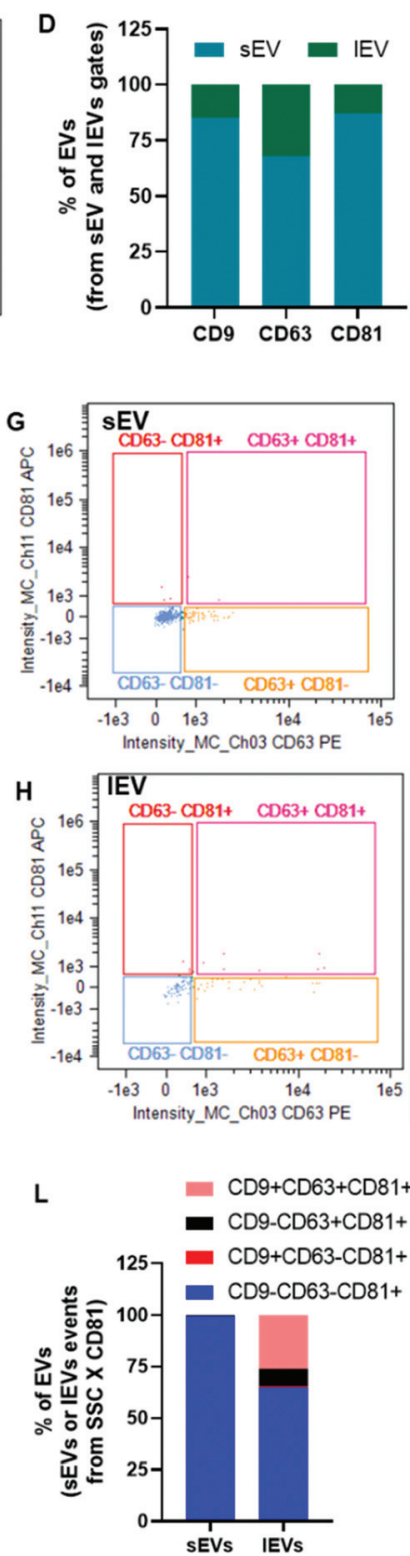

Fig. 3 EV analysis by multispectral imaging flow cytometry. Biparametric dot plots of the side scatter intensity (SSC) versus fluorescence intensity corresponding to CD9 FITC, CD63 PE and CD81 APC (A, B and C, respectively). The percentages of small EVs (sEVs) and large EVs (IEVs) considering the sum of them for each of these three biparametric dot plots were calculated (D). Images were acquired for single EVs in the bright field, fluorescence channels for FITC (CD9), PE (CD63) and APC (CD81) as well as in the dark field for sEVs (E) and IEVs (F). The events in the EV gates for the SSC versus CD9 FITC fluorescence intensity were further plotted for CD81 APC versus CD63 PE fluorescence intensities to investigate single, double and triple positiveness for sEVs (G) and IEVs (H). The quantitative bar graph of this plot indicated the \% of single CD9+, double CD81+CD9+, double CD63+CD9+ and triple CD63+CD81+CD9+ (I). The events in the EV gate for the SSC versus CD81 APC fluorescence intensity were further plotted for CD63 PE versus CD9 FITC intensities to investigate single, double and triple positiveness for sEVs (J) and IEVs (K). The quantitative bar graph of this plot indicated the percentages of single CD81+, double CD63+CD81+, double CD9+CD81+ and triple CD9+CD63+CD81+ (L).

\section{Radiological evaluation}

One pig (control group) was excluded from the radiological analysis because the stricture reached the cardia and it was not possible to measure the post-stenotic esophagus diameter. Esophagography analysis indicated that the degree of stricture was significantly lower in the $\mathrm{EV}+$ gel group $(67.9 \%)$ in comparison with the control group $(92.5 \%)(p=0.04)$. This rep- resents the primary outcome of the in vivo study. The difference was not significant when comparing the gel group $(83.4 \%)$ to control or to the EV + gel group (Fig. 6B and C).

\section{Histological evaluation}

Histological analysis for the $\mathrm{EV}+$ gel group evidenced that (i) the minimal length of muscularis mucosae was signifi- 
A

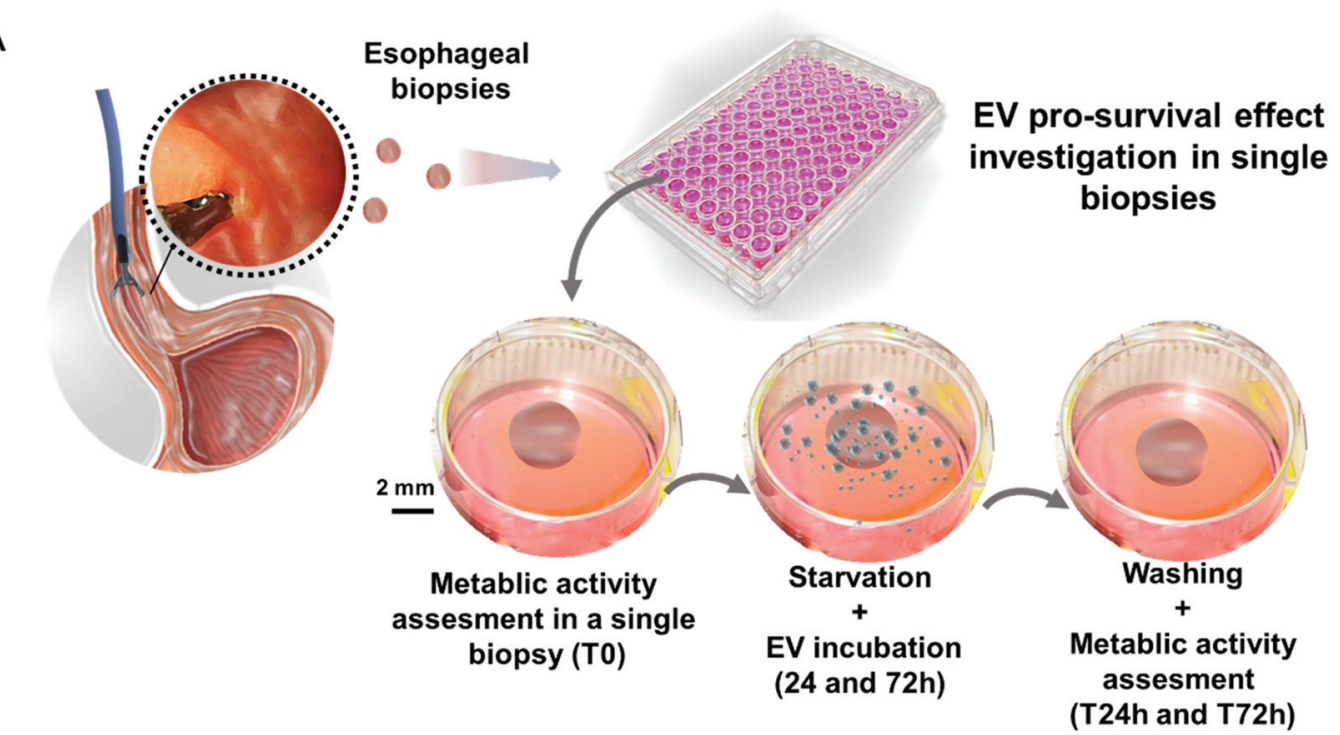

B

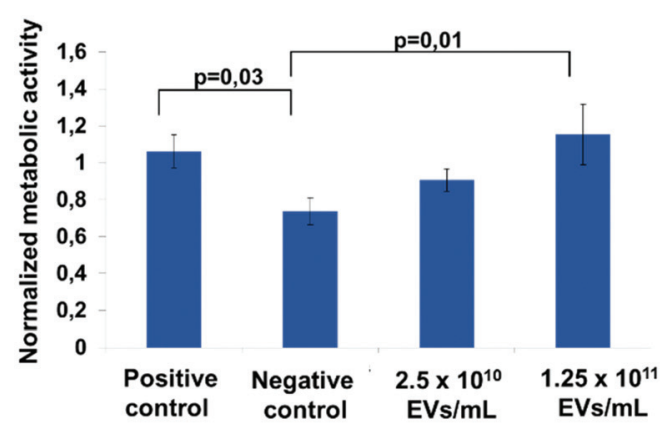

C

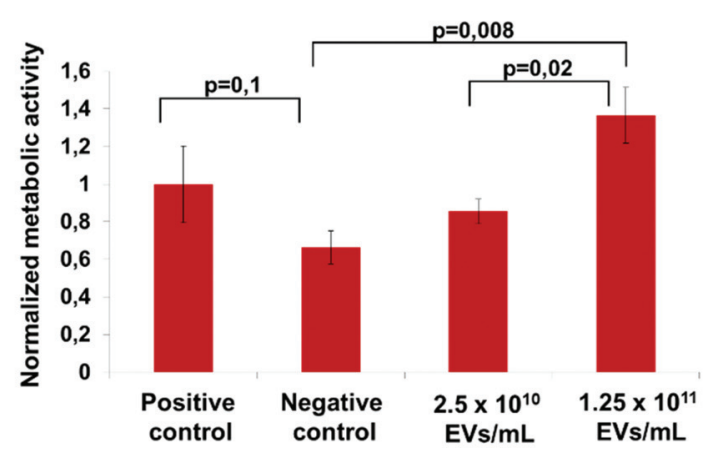

Fig. 4 The potency evaluation ex vivo of ADSC EVs by a single-biopsy pro-survival test. Schematic representation of the assay from biopsy sampling of the esophagus from pigs by endoscopy to single biopsy metabolic activity assessment in individual wells under starvation before and after incubation with ADSC EVs (A). Metabolic activity of single biopsies normalized to T0 values after incubation with $2.5 \times 10^{10}$ or $1.25 \times 10^{11} \mathrm{ADSC}^{\mathrm{E}} \mathrm{Es} \mathrm{mL}^{-1}$ under starvation in comparison with a negative (starvation) control and a positive (complete medium) control at $24 \mathrm{~h}$ (B) and $72 \mathrm{~h} \mathrm{(C)}$.

cantly higher compared to that of the control group (Fig. 6D); (ii) there was a significantly smaller fibrosis area compared to the gel group (Fig. 6E) (statistically significant difference compared to the control group was not observed in a sample of 6 animals per group due to the wide dispersion of control values); (iii) there was superficial fibrosis without infiltration into the muscularis propria in comparison with the control group and also to the gel one (both control and gel groups feature thick fibrosis with a dilapidated fibrosis infiltration within the muscularis propria) (Fig. 6F).

Histological analysis is further reported in ESI Table $4 . \dagger$ There was no significant difference between the groups in terms of the maximal length of defect of epithelium neither in terms of the maximal thickness of fibrosis. In the $\mathrm{EV}+$ gel group, the length of muscularis mucosae was higher when compared to those in the gel and control groups.

\section{Discussion}

We evaluated herein an innovative minimally invasive nanotherapy to prevent esophageal stricture in pigs based on endoscopic injection of ADSC EVs and delivery in a thermoresponsive PF-127 gel. Neither EV-based esophageal stricture prevention nor the thermo-actuated delivery strategy has been explored before for this disorder.

Thermoresponsive injectable hydrogels offer the advantage of administration in a liquid state at a temperature below $19{ }^{\circ} \mathrm{C}$. This facilitates the delivery to the gastrointestinal mucosal by using an appropriate injection device; in our case, a catheter is introduced via an endoscope. Such an administration strategy allowed us to cover the wide extent of the resection lesion with EVs embedded into the PF-127 gel. Once the composition comes into contact with the resection lesion at body temperature, there is gel transition characterized by a viscosity increase. This has the advantage of enabling the compo- 


\section{A}

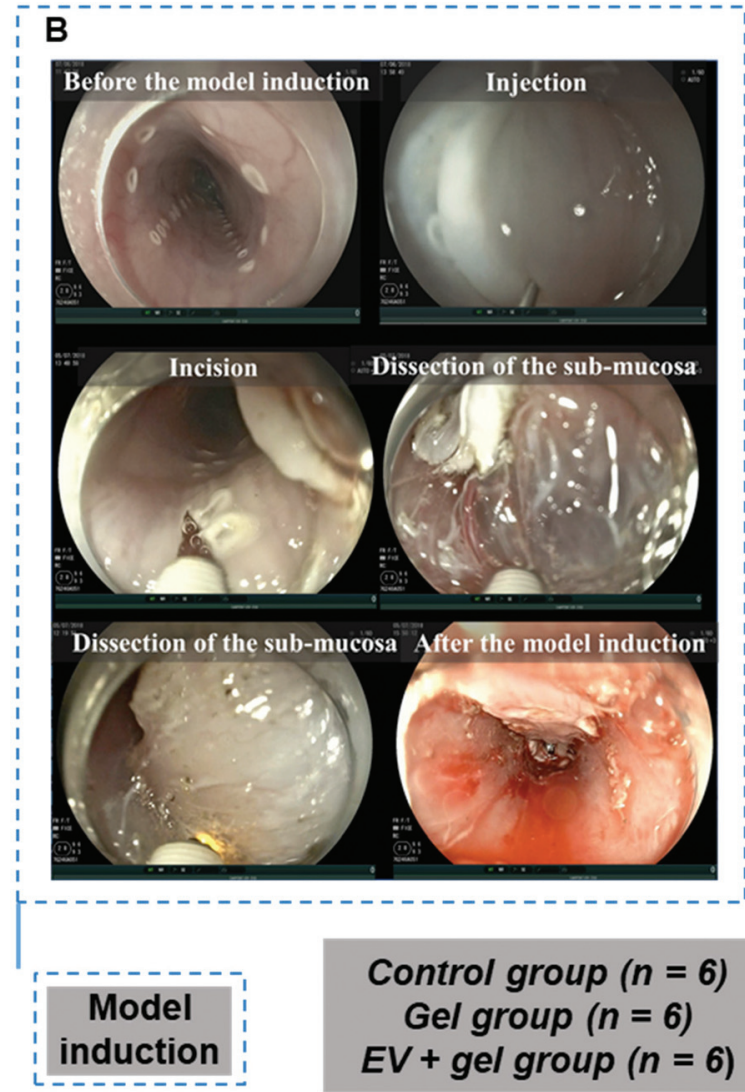

;----------

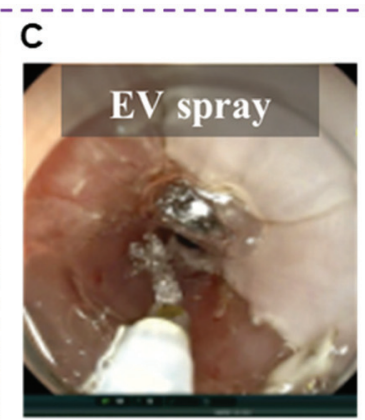

EV injection

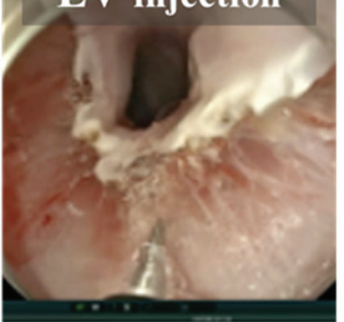

EVs + gel application
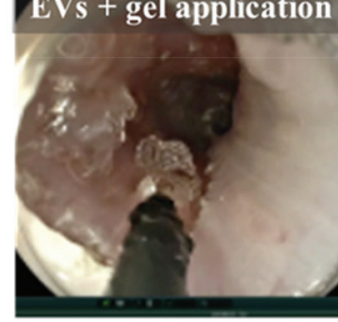

\section{Therapy follow-up (pre-clinical analysis, endoscopy, radiology and histology)}

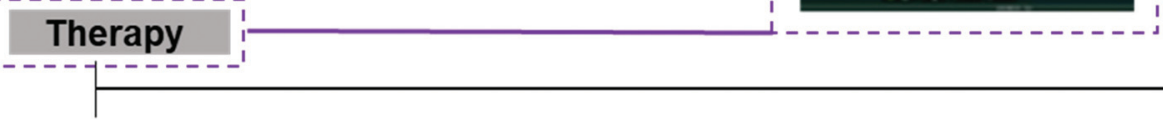

D0

D21

Fig. 5 Design of the in vivo study and overview of the main steps. In vivo study timeline and sample size per group (A). Endoscopic procedure for the esophageal stricture model induction from saline injection and incision to dissection (B). Endoscopic views of the therapy procedure for the EV + gel group in three steps: (i) ADSC EV spraying, (ii) ADSC EV injection and (iii) application of the ADSC EVs plus gel in the esophageal resection lesions (C). Note that for the gel group, there was only this third step with the application of the gel alone.

Table 1 Clinical and endoscopic evaluation parameters for control, gel and EV + gel groups

\begin{tabular}{|c|c|c|c|c|}
\hline Parameter & Control group $(n=6)$ & Gel group $(n=6)$ & $\mathrm{EV}+$ gel group $(n=6)$ & $p$ \\
\hline Cases with feasible passage of the endoscope (\%) & $0(0 \%)$ & $0(0 \%)$ & $1(16.67 \%)$ & 1 (NS) \\
\hline
\end{tabular}

sition to remain on the surface of the affected area facilitating the contact and the residence time, transiently retaining EVs in the site of interest. Endoscopic administration of EVs into the PF-127 gel was previously tested in the therapy of esophageal fistulas by our team. ${ }^{38}$ In that study, we evidenced by rheological analysis that the integration of EVs into the PF-127 gel did not alter its thermoresponsive properties. The EV integration into the gel also seemed to have a minor impact on the integrity of EVs. Besides, we evidenced that the EV release was expected to be driven by the saliva flow exerting a dilution effect. We formerly showed that gel dilution to a concentration exceeding the threshold of about $13 \%$ notably induced a viscosity decrease, releasing EVs. ${ }^{38}$ Indeed, NTA experiments indicated that EVs were entrapped in the PF-127 gel when its concentration was $20 \%$, which was evidenced by the absence of Brownian motion. Such a trapping effect was reduced by gel dilution by a factor 2 . To a greater extent, dilution by a factor of 10 intensively released EVs featuring Brownian motion. The EV release after gel dilution is expected to render EVs able to interact with recipient cells/tissues. $^{38}$

In addition to the ADSC EV delivery embedded into the gel, a previous step was the administration of ADSC EVs in saline 
A

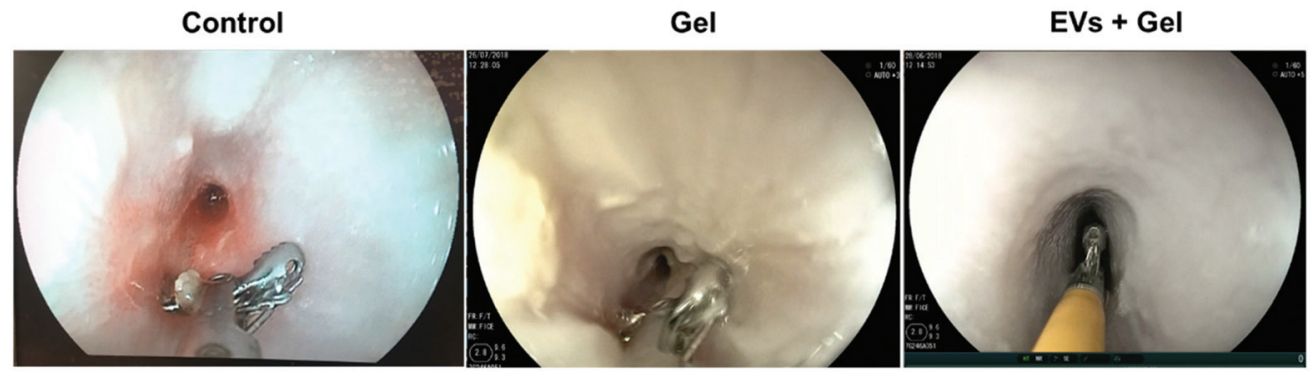

B

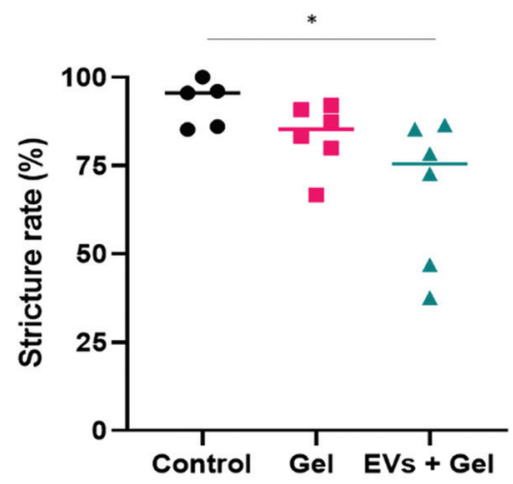

D

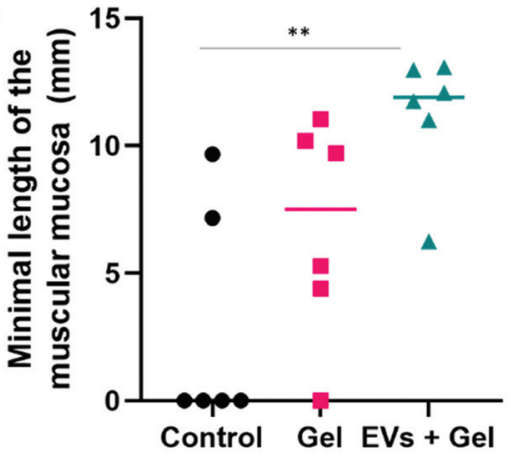

E

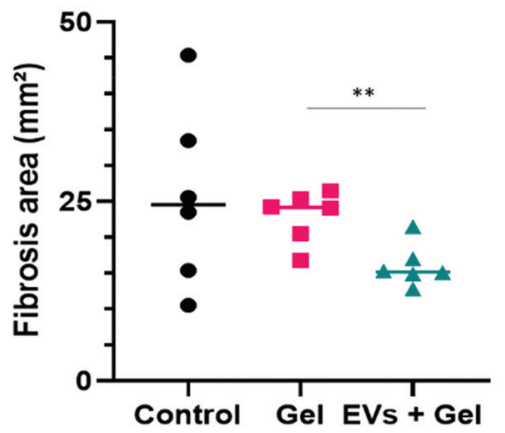

C
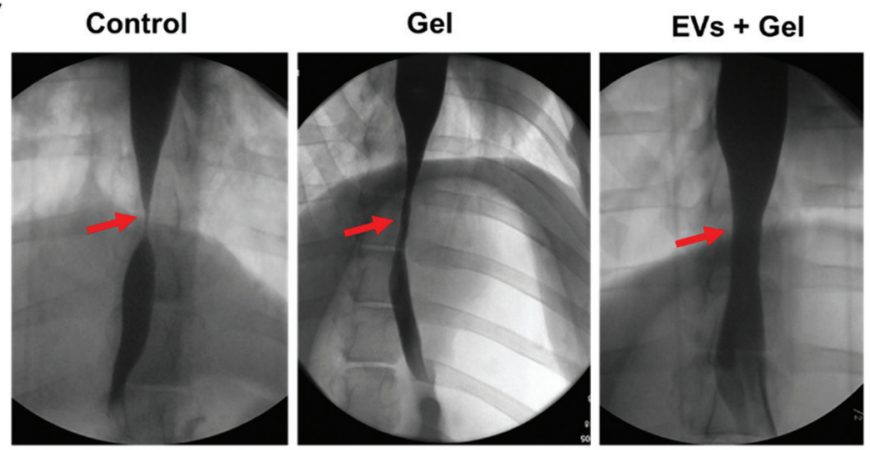

$\mathbf{F}$
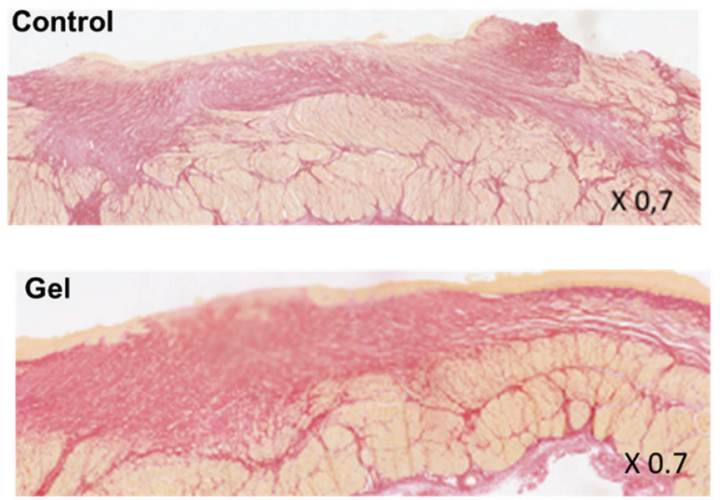

$\mathrm{EVs}+\mathrm{Gel}$
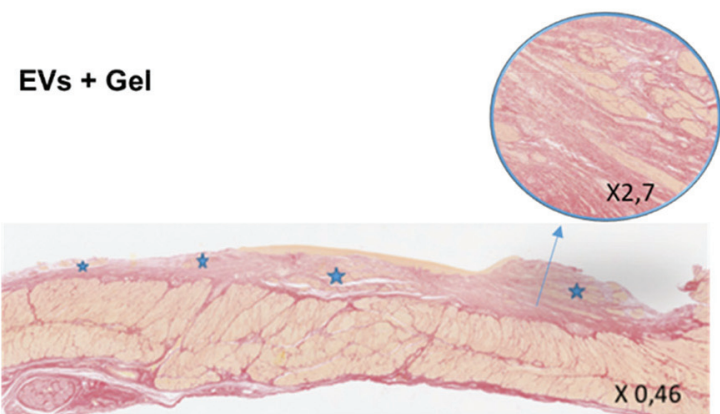

Fig. 6 The potency evaluation in vivo for ADSC EVs by a multi-modal approach in pigs treated with the gel or ADSC EVs + gel in comparison with untreated negative control. Therapy follow-up by endoscopic evaluation assessing the feasibility of an open biopsy forceps (7 mm in diameter) passage through the esophageal stricture (A). Therapy follow-up by radiologic evaluation assessing the stricture rate (B) calculated from esophagography (C). The minimal length of the muscular mucosa (D) and the fibrosis area (E) quantified by histological analysis. Histological sections, colored by Sirius red for fibrosis evaluation. The upper, middle and bottom panels correspond to histological sections from the control, gel and EV + gel groups (respectively). For the latter, the inset shows a zoom-in of the muscularis mucosa and stars correspond to areas where this layer is preserved (F).

over the entire surface of the esophageal resection lesion using a spray catheter. This thin aqueous layer enabled a fraction of EVs to be in direct contact with the tissue in complement to the thicker gel layer embedding EVs administered later.
Additionally, EVs were injected into the edges of lesions in order to maximize the healing effect. Indeed, "leader" cells and follower ones originating from the lesion borders play an important role in wound healing. ${ }^{41}$ We consider that EV 
administration at this strategic site may be beneficial for favoring the healing process. In this regard, it has been reported that EV trails left behind the migrating leader cells would be able to promote chemotaxis of the follower ones. ${ }^{42,43}$

On day 21 post-therapy, a multimodal follow-up was performed. Endoscopy, radiology and histology data consistently indicated a significantly reduced stricture rate and fibrosis area and an increased mucosal muscle width by the combined action of EVs and the thermoresponsive hydrogel. The therapeutic outcome with PF-127 seemed better than that with the control group. Indeed, there was a trend towards the reduction of the stricture rate and an increase in the minimal length of the muscular mucosa compared to the control. However, in both cases, a statistically significant difference was not reached, possibly due to the number of animals per group. Previous studies reported that the topical application of the PF-127 gel promoted cutaneous wound healing in rats and increased microvessel density. ${ }^{37}$ In particular, increased expression of the vascular endothelial growth factor (VEGF) and transforming growth factor-beta1 (TGF- $\beta 1$ ) was observed. These effectors probably played an important role in the recruitment of the inflammatory cells, angiogenesis and granulation tissue formation since histopathological analyses revealed more leukocyte infiltration accompanied by wellformed granulation tissue and fibroblast proliferation in the group treated with the PF-127 gel. ${ }^{37}$ A moderate therapeutic effect in fistula healing was also evidenced in pigs after the administration of the gel alone, including a trend towards the reduction of the inflammation score compared to control. ${ }^{38}$ Therefore, the PF-127 gel may be considered more than a carrier vehicle, contributing by itself to some extent to the therapeutic effect observed in the pig stricture model reported herein. Importantly, the PF-127 gel effect in the prevention of esophageal stricture was markedly enhanced by the combination with ADSC EVs. These EVs are expected to carry proregenerative signaling to help in restoring tissue homeostasis. Importantly, in an ex vivo setting, we evidenced the pro-survival effect of our ADSC EVs in esophageal tissue single biopsies under starvation. To our knowledge, this is the first time that pro-survival EV potency is assessed on a single biopsy basis. We had previously performed pro-survival potency tests in monolayers of cells isolated from esophageal biopsies following an enzymatic tissue dissociation treatment to obtain individual cells. ${ }^{38}$ We are aware of all the limitations of the monolayer system, and we believe that alternative approaches should be investigated to bridge the gap between in vitro and in vivo studies. Our single biopsy strategy herein may be of interest in this regard.

Concerning the obtained in vivo data, we showed that the degree of esophageal stricture was lower in the EV + gel group compared with those in the gel and control ones in this porcine model of esophageal stricture induced by extensive ESD. The endoscopic evaluation indicated a less severe stricture in the $\mathrm{EV}+$ gel group. In the control group, it is interesting to note the occurrence of infiltrative fibrosis reaching the muscularis propria. This is in agreement with a study evaluat- ing the role of fibrosis after mucosectomy in dogs, in which the fibrosis-mediated destruction of the muscularis mucosae was shown. ${ }^{24}$ In the same study, fibrosis induced atrophy of the muscularis propria fibers. EVs plus gel induced an antifibrotic effect, also inhibiting muscularis mucosae atrophy. Indeed, animals from the EV + gel group featured a higher length of muscularis mucosae compared to the other groups. The fibrosis was more superficial and did not invade the muscularis propria in the EV + gel group. These results indicating the combined action of ADSC EVs plus PF-127 gel leading to a morphological tissue switch towards a less fibrotic and less atrophic state in the muscularis mucosae are very promising. Previous reports evidenced the anti-fibrotic action of ADSC EVs, including in ureteral stricture rat models. ${ }^{44}$

Our study concerned a single EV dose administration. Other authors showed the interest of multiple-dose administration in order to obtain an enhanced therapeutic effect. ${ }^{7}$ Undoubtedly, the possibility of obtaining greater effects by repeated doses may be considered of interest. In particular, limitations in the gel residence time due to esophageal peristalsis, swallowing movements and saliva dilution could be counterbalanced by repeated applications.

In this study, we used EVs from porcine ADSCs. These cells have many advantages, and some of them are their ease of harvesting without an invasive intervention compared to SCs from bone marrow and the highest SC yield obtained. ${ }^{45}$ As far as we know, this is the first time that ADSC-EVs have been investigated to prevent esophageal strictures as an alternative to cell therapy. SCs, as whole cells, administered via an endoscopic procedure have been previously investigated for this therapeutic indication by others in a canine ESD lesion model. ${ }^{46}$ In a previous report of our team, SCs were organized in double cell sheets, and we showed a significant improvement in the rate of esophageal strictures in pigs. ${ }^{32}$ However, the manufacturing of cell sheets requires particular expertise and needs to be planned several days before resection. ${ }^{47}$ Several advantages emerge when using EV-based nano-therapies with respect to the whole cells, among which EVs are safer and easier to handle due to their smaller size and the absence of the nucleus, thus avoiding self-replication and highly reducing the cell endogenous tumorigenic potential. ${ }^{12}$ Moreover, EVs, as carriers for bioactive molecules, may be exploited to overcome tissue barriers to reach specific cell populations. Additionally, EVs can be stored in a freezer for several months, offering a banking possibility. ${ }^{13}$ Besides, it is interesting to note that allogeneic transplantation of ADSCs is well-tolerated because these cells have an immunosuppressive role, they do not express class II MHC or co-activating molecules of lymphocyte response and EVs from MSC probably have the same immunotolerance status. ${ }^{48}$ This immune-privileged status of ADSC EVs in an allogeneic setting and the banking possibilities may open the way towards an "off-the-shelf" therapeutic modality.

ADSC EVs were produced herein in a scalable and high yield approach in bioreactors, which contributes to the translational potential of our investigation. Compared to the starvation approach, the turbulence approach in $4 \mathrm{~h}$ enabled a 
more than 5-fold increase in the EV production yield compared to $48 \mathrm{~h}$ of starvation and more than a 4 -fold increase in $72 \mathrm{~h}$ of starvation, which means an 18 times shorter time for the latter. We previously evidenced that the turbulence approach was scalable based on turbulence physical laws and enabled the production of EVs featuring markers, molecular content and potency equivalent to state-of-the-art starvation EVs. ${ }^{39}$ Advantageously, our turbulence approach is directly integrated into the scalable cell culture process in stirred tank bioreactors. Its originality is to go beyond the bioreactor use as a cell culture device. Indeed, we use the bioreactor stirring system in a dual manner, for homogenization (its primary purpose) and for triggering massive EV production via the generated turbulence shear stress. For instance, the required dose to treat 2 pigs was obtained from 250 million ADSCs in $1 \mathrm{~L}$ bioreactors with a volume of $380 \mathrm{~mL}$ of medium. The high-yield turbulence approach enabled us to produce EVs efficiently in the human dose range in a sufficient amount to be tested in a clinically relevant setting using large animals.

\section{Conclusions}

One of the major contributions of our study is that we investigated the proposed nano-therapy in a porcine model, allowing us to ascertain esophageal stricture disease, administration modalities as well as the required therapeutic doses relevant to humans in a translational approach. Another strength of our study is the association of nanoscale EVs with a thermoresponsive biomaterial constituted of nano-micelles ${ }^{36,38}$ to prevent esophageal strictures. Our results strengthen the view that a combination of EVs and a biomaterial may improve the biomaterial therapeutic effect while facilitating EV administration. We have shown for the first time that ADSC EV-based treatment combined with endoscopic injection of a thermoresponsive gel of Pluronic ${ }^{\circledR}$ F-127 prevented the esophageal stricture in a porcine model by an anti-fibrotic and regenerative effect on the muscular mucosae. The confirmation of this preventive effect in a clinical study could provide real hope for patients tackling current unmet needs.

\section{Author contributions}

Conception, design, and study supervision: Amanda Karine Andriola Silva and Gabriel Rahmi. Acquisition of data: Elise Coffin, Guillaume Perrod, Iris Marangon, Max Piffoux, Alice Grangier, Arthur Berger, Imane Boucenna, Leila M'Harzi, Jessica Assouline, and Thierry Lecomte. Analysis and interpretation of data: Elise Coffin, Amanda Karine Andriola Silva, Gabriel Rahmi, Olivier Clément, and Christophe Cellier. Writing, review, and/or revision of the manuscript: Amanda Karine Andriola Silva, Gabriel Rahmi, Claire Wilhelm, Florence Gazeau, and Imane Boucenna.

\section{Conflicts of interest}

Florence Gazeau, Amanda Karine Andriola Silva, Claire Wilhelm and Gabriel Rahmi are co-founders of the spin-off Evora Biosciences. Amanda Karine Andriola Silva and Claire Wilhelm are co-founders of the spin-off EverZom. The other authors have no conflicts to declare.

\section{Acknowledgements}

This work was funded by the Centre national de la recherche scientifique (CNRS) via the PEPS INSIS program "Sciences de l'ingénierie pour la santé pour accompagner des projets translationnels". This work was also supported by the Region Ile-deFrance via the DIM ELICIT (EVORTEX project). The authors thank Jean-Michel Guigner from the facility "Plateforme Microscopie électronique en transmission de l'IMPMC", LUMIC-Sorbonne, for cryo-TEM analysis. This study was supported by the IdEx Université de Paris, ANR-18-IDEX-0001 and by the Region Ile de France under the convention SESAME 2019 - IVETh (no. EX047011).

\section{References}

1 M. Mathieu, L. Martin-Jaular, G. Lavieu and C. Théry, Nat. Cell Biol., 2019, 21, 9-17.

2 O. G. De Jong, B. W. Van Balkom, R. M. Schiffelers, C. V. Bouten and M. C. Verhaar, Front. Immunol., 2014, 5, 608.

3 L. A. Reis, F. T. Borges, M. J. Simões, A. A. Borges, R. Sinigaglia-Coimbra and N. Schor, PLoS One, 2012, 7, e44092.

4 Y. Zhou, H. Xu, W. Xu, B. Wang, H. Wu, Y. Tao, B. Zhang, M. Wang, F. Mao and Y. Yan, Stem Cell Res. Ther., 2013, 4, 1-13.

5 X. Liang, Y. Ding, Y. Zhang, H.-F. Tse and Q. Lian, Cell Transplant., 2014, 23, 1045-1059.

6 R. C. Lai, F. Arslan, M. M. Lee, N. S. K. Sze, A. Choo, T. S. Chen, M. Salto-Tellez, L. Timmers, C. N. Lee and R. M. El Oakley, Stem Cell Res., 2010, 4, 214-222.

7 S. Bruno, C. Grange, F. Collino, M. C. Deregibus, V. Cantaluppi, L. Biancone, C. Tetta and G. Camussi, PLoS One, 2012, 7, e33115.

8 T. Li, Y. Yan, B. Wang, H. Qian, X. Zhang, L. Shen, M. Wang, Y. Zhou, W. Zhu and W. Li, Stem Cells Dev., 2013, 22, 845-854.

9 B. Zhang, M. Wang, A. Gong, X. Zhang, X. Wu, Y. Zhu, H. Shi, L. Wu, W. Zhu and H. Qian, Stem Cells, 2015, 33, 2158-2168.

10 Q. Long, D. Upadhya, B. Hattiangady, D.-K. Kim, S. Y. An, B. Shuai, D. J. Prockop and A. K. Shetty, Proc. Natl. Acad. Sci. U. S. A., 2017, 114, E3536-E3545. 
11 H. Cao, Y. Cheng, H. Gao, J. Zhuang, W. Zhang, Q. Bian, F. Wang, Y. Du, Z. Li and D. Kong, ACS Nano, 2020, 14, 4014-4026.

12 S. E. Andaloussi, I. Mäger, X. O. Breakefield and M. J. Wood, Nat. Rev. Drug Discovery, 2013, 12, 347-357.

13 R. C. Lai, T. S. Chen and S. K. Lim, Regen. Med., 2011, 6, 481-492.

14 D. Allan, A. Tieu, M. Lalu and D. Burger, Stem Cells Transl. Med., 2020, 9, 39-46.

15 A. Tieu, M. M. Lalu, M. Slobodian, C. Gnyra, D. A. Fergusson, J. Montroy, D. Burger, D. J. Stewart and D. S. Allan, ACS Nano, 2020, 14, 9728-9743.

16 C. Fitzmaurice, D. Dicker, A. Pain, H. Hamavid, M. MoradiLakeh, M. F. MacIntyre, C. Allen, G. Hansen, R. Woodbrook and C. Wolfe, JAMA Oncol., 2015, 1, 505-527.

17 K. M. Sjoquist, B. H. Burmeister, B. M. Smithers, J. R. Zalcberg, R. J. Simes, A. Barbour, V. Gebski and Australasian Gastro-Intestinal Trials Group, Lancet Oncol., 2011, 12, 681-692.

18 P. Pimentel-Nunes, M. Dinis-Ribeiro, T. Ponchon, A. Repici, M. Vieth, A. De Ceglie, A. Amato, F. Berr, P. Bhandari and A. Bialek, Endoscopy, 2015, 47, 829-854.

19 S. Abe, P. G. Iyer, I. Oda, N. Kanai and Y. Saito, Gastrointest. Endosc., 2017, 86, 779-791.

20 J. S. Kim, B.-W. Kim and I.-S. Shin, Dig. Dis. Sci., 2014, 59, 1862-1869.

21 S. Ono, M. Fujishiro, K. Niimi, O. Goto, S. Kodashima, N. Yamamichi and M. Omata, Endoscopy, 2009, 41, 661665.

22 W. Peng, S. Tan, Y. Ren, H. Li, Y. Peng, X. Fu and X. Tang, J. Cardiothorac. Surg., 2020, 15, 33.

23 R. Kramann, D. P. DiRocco and B. D. Humphreys, J. Pathol., 2013, 231, 273-289.

24 M. Honda, T. Nakamura, Y. Hori, Y. Shionoya, A. Nakada, T. Sato, K. Yamamoto, T. Kobayashi, H. Shimada and N. Kida, Endoscopy, 2010, 42, 1092-1095.

25 J. J. Lian, L. L. Ma, J. W. Hu, S. Y. Chen, W. Z. Qin, M. D. Xu, P. H. Zhou and L. Q. Yao, J. Dig. Dis., 2014, 15, 224-229.

26 H. Takahashi, Y. Arimura, S. Okahara, S. Uchida, S. Ishigaki, H. Tsukagoshi, Y. Shinomura and M. Hosokawa, Endoscopy, 2011, 43, 184-189.

27 J. Wen, Z. Lu and Q. Liu, Gastroenterol. Res. Pract., 2014, 2014, 457101.

28 N. Hanaoka, R. Ishihara, Y. Takeuchi, N. Uedo, K. Higashino, T. Ohta, H. Kanzaki, M. Hanafusa, K. Nagai and F. Matsui, Endoscopy, 2012, 44, 1007-1011.

29 E. Rajan, C. Gostout, A. Feitoza, L. Herman, M. Knipschield, L. Burgart, S. Chung, P. Cotton, R. Hawes and A. Kalloo, Endoscopy, 2005, 37, 1111-1115.
30 I. Bon, R. Bartolí and V. Lorenzo-Zúñiga, World J. Gastroenterol., 2017, 23, 3761.

31 T. Ohki, M. Yamato, M. Ota, R. Takagi, D. Murakami, M. Kondo, R. Sasaki, H. Namiki, T. Okano and M. Yamamoto, Gastroenterology, 2012, 143, 582-588.

32 G. Perrod, G. Rahmi, L. Pidial, S. Camilleri, A. Bellucci, A. Casanova, T. Viel, B. Tavitian, C. Cellier and O. Clement, PLoS One, 2016, 11, e0148249.

33 L. Casteilla, V. Planat-Benard, P. Laharrague and B. Cousin, World J. Stem Cells, 2011, 3, 25.

34 S. Rani, A. E. Ryan, M. D. Griffin and T. Ritter, Mol. Ther., 2015, 23, 812-823.

35 G. Dumortier, J. L. Grossiord, F. Agnely and J. C. Chaumeil, Pharm. Res., 2006, 23, 2709-2728.

36 A. Berger, I. Araújo-Filho, M. Piffoux, A. Nicolás-Boluda, A. Grangier, I. Boucenna, C. C. Real, F. L. N. Marques, D. de Paula Faria, A. C. M. do Rego, C. Broudin, F. Gazeau, C. Wilhelm, O. Clément, C. Cellier, C. A. Buchpiguel, G. Rahmi and A. K. A. Silva, Nanoscale, 2021, 13, 218-232.

37 V. Kant, A. Gopal, D. Kumar, A. Gopalkrishnan, N. N. Pathak, N. P. Kurade, S. K. Tandan and D. Kumar, Acta Histochem., 2014, 116, 5-13.

38 A. K. Silva, S. Perretta, G. Perrod, L. Pidial, V. Lindner, F. Carn, S. Lemieux, D. Alloyeau, I. Boucenna, P. Menasché, et al., ACS Nano, 2018, 12, 9800-9814.

39 M. Piffoux, A. K. A. Silva, O.-W. Merten, C. Wilhelm and F. Gazeau, patent application WO2019002608, 2019.

40 M. H. Mellow and H. Pinkas, Arch. Intern. Med., 1985, 145, 1443-1446.

41 T. Omelchenko, J. Vasiliev, I. Gelfand, H. Feder and E. Bonder, Proc. Natl. Acad. Sci. U. S. A., 2003, 100, 1078810793.

42 B. H. Sung, T. Ketova, D. Hoshino, A. Zijlstra and A. M. Weaver, Nat. Commun., 2015, 6, 1-14.

43 B. H. Sung, A. von Lersner, J. Guerrero, E. S. Krystofiak, D. Inman, R. Pelletier, A. Zijlstra, S. M. Ponik and A. M. Weaver, Nat. Commun., 2020, 11, 1-15.

44 J. Luo, S. Zhao, J. Wang, L. Luo, E. Li, Z. Zhu, Y. Liu, R. Kang and Z. Zhao, J. Cell. Mol. Med., 2018, 22, 44494459.

45 M. Strioga, S. Viswanathan, A. Darinskas, O. Slaby and J. Michalek, Stem Cells Dev., 2012, 21, 2724-2752.

46 M. Honda, Y. Hori, A. Nakada, M. Uji, Y. Nishizawa, K. Yamamoto, T. Kobayashi, H. Shimada, N. Kida and T. Sato, Gastrointest. Endosc., 2011, 73, 777-784.

47 N. Yamaguchi, H. Isomoto, S. Kobayashi, N. Kanai, K. Kanetaka, Y. Sakai, Y. Kasai, R. Takagi, T. Ohki and H. Fukuda, Sci. Rep., 2017, 7, 1-12.

48 C.-S. Lin, G. Lin and T. F. Lue, Stem Cells Dev., 2012, 21, $2770-2778$ 